
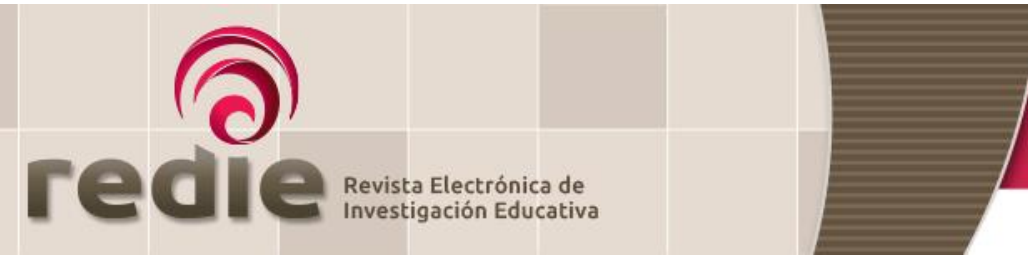

\title{
Teoría del capitalismo académico en los estudios de educación superior ${ }^{1}$
}

\author{
José Joaquín Brunner (1) josejoaquin.brunner@gmail.com \\ Julio Roberto Labraña Vargas (1) jlabrana@ug.uchile.cl \\ Francisco Ganga (2) fganga@ulagos.cl \\ Emilio Rodríguez-Ponce (3) emilior.rodriguez.ponce@gmail.com \\ (1) Universidad Diego Portales \\ (2) Universidad de Los Lagos \\ (3) Universidad de Tarapacá \\ (Recibido: 8 de julio de 2019; Aceptado para su publicación: 14 de octubre de 2019)
}

Cómo citar: Brunner, J. J., Labraña, J. R., Ganga, F. y Rodríguez-Ponce, E. (2019). Teoría del capitalismo académico en los estudios de educación superior. Revista Electrónica de Investigación Educativa, 21, e33, 1-13. doi:10.24320/redie.2019.21.e33.3181

\section{Resumen}

Este artículo examina la difusión y recepción del enfoque angloamericano del academic capitalism en el campo internacional de estudios de la educación superior. En primer lugar se identifican los documentos indexados por Google Scholar cuyos títulos contiene las palabras "academic capitalism" en inglés, español y portugués con el objetivo de conocer, mediante contabilidad de citas, su impacto en la literatura; enseguida se analizan las características conceptuales y contenidos de los documentos. Los resultados muestran diferencias en el uso de la teoría del academic capitalism. Mientras en la literatura angloamericana se utiliza con propósito de análisis empírico e interpretación teórica, en el caso del español y del portugués prima una orientación hacia la descripción y el cuestionamiento de los sistemas de educación superior latinoamericanos en su estado actual, sin considerar los alcances conceptuales de la teoría. Se concluye presentando tres desafíos de investigación.

Palabras clave: Capitalismo académico, educación superior, teoría de la educación, bibliometría.

\section{Introducción}

La teoría del academic capitalism (AC) comienza a ser estudiada en el campo latinoamericano de estudios de educación superior (Brunner, 2017; Fernández, 2009; Montes y Mendoza, 2018); sin embargo, no se ha abordado el modo en el cual esta teoría ha sido recibida y aplicada en América Latina. Llenar tal vacío es el objetivo del presente artículo.

La respuesta se elabora a partir del análisis de la literatura especializada, particularmente de la forma como aquella recepción opera en la periferia (Guzmán-Valenzuela y Barnett, 2019); en este caso, la emergente área latinoamericana de estudios de la educación superior (Brunner, 2009; GuzmánValenzuela y Gómez, 2019; Ordorika y Rodríguez-Gómez, 2018). Por lo pronto, la circulación de la teoría que nos ocupa se encuentra estructurada por la existencia de una asimetría entre los polos emisor y receptor. No cabe duda de dónde se origina la teoría, ni respecto a la densidad institucional del campo de estudios en uno y otro polo, ni del carácter dependiente de teorías importadas que exhibe el campo localnacional-regional, ni de las asimetrías de poder político, económico y de prestigio entre el centro productor y el margen consumidor. Estamos, pues, frente a una recepción que ocurre "a lo largo de una

\footnotetext{
${ }^{1}$ Proyecto FONDECYT N 1180746 - Sistema universitario chileno: gobernanza del capitalismo académico y calidad de las instituciones.
} 
línea divisoria jerárquica que corre de arriba hacia abajo entre lugares prestigiosos de producción de teoría y la periferia" (Keim, 2014, p. 8).

Para avanzar en el estudio del proceso de recepción se procederá, en primer lugar, a establecer una muestra de la literatura dedicada específicamente al conglomerado conceptual del capitalismo académico en idioma inglés y en español y portugués. Enseguida, los respectivos documentos se someten a revisión, luego se presenta el resultado de dicha revisión, contrastando aspectos cuantitativos y sustantivos de las dos muestras de literatura empleada. Finalmente, los resultados obtenidos se analizan desde el punto de vista de la recepción y el uso de la teoría en el contexto latinoamericano. La conclusión formula algunas sugerencias que se espera estimulen el análisis del capitalismo académico en América Latina.

\subsection{Capitalismo académico: aproximación a sus orígenes}

El enfoque del AC es un esfuerzo conceptual que desde fines del siglo pasado busca explicar las transformaciones experimentadas por la educación superior en los países desarrollados de alto ingreso del mundo anglosajón. Fue elaborado inicialmente por un grupo de autores de la Universidad de Arizona: Sheila Slaughter, socióloga; Larry L. Leslie, economista, y Gary Rhoades, sociólogo. Las dos obras clave que inauguran este enfoque, una de Slaughter y Leslie (1997), la otra de Slaughter y Rhoades (2004), reclaman para sí, explícitamente, el carácter teórico de ese esfuerzo. El enfoque del Ac nace como un intento por describir sistemáticamente las circunstancias y las políticas que dan comienzo a un período de mercantilización de las universidades de aquellos países, particularmente los Estados Unidos de América. Se refiere, por tanto, a la actividad universitaria ligada a los mercados; al ingreso de recursos obtenidos por vía comercial y, en general, a la valorización económica de los productos y servicios de conocimiento (Brunner et al., 2019). Su posterior desarrollo consistiría en una extensión y profundización de dichos procesos.

En términos de los autores involucrados con la teoría del Ac, el concepto referido a este fenómeno es convergente, aunque las palabras clave que lo acompañan varían en cada caso. Originalmente, Slaughter y Leslie (1997) ofrecieron una definición estrechamente asociada con los juegos del mercado. Sostienen que el capitalismo académico trata de los comportamientos de mercado y de tipo de mercado de las universidades y sus profesores, tales como competencia por dinero proveniente de diferentes fuentes, colaboración universidad-industria, inversión en spin-offs, aranceles y tasas pagados por los estudiantes y otras actividades dirigidas a generar recursos.

Algunos años después, los fundadores de la teoría adoptaron una visión más amplia, definiendo al Ac en relación con circuitos de conocimiento, redes de actores, organismos intersticiales y capacidades expandidas de gestión de las universidades públicas al servicio de su integración en la nueva economía. Al mismo tiempo, avanzaron la hipótesis de que el AC va aparejado de la agencia de hacedores de políticas, administradores, profesores y estudiantes que, desde dentro de la universidad, utilizan recursos del Estado para aprovechar esas oportunidades de integración, redefiniendo el carácter de la institución en este proceso. Adicionalmente, los autores plantean una segunda hipótesis que en su momento representó un enriquecimiento de la teoría del AC, la cual es sostiene que éste conduce a la aparición de un nuevo régimen de conocimiento y aprendizaje, el que de allí en adelante moldearía las actividades de las universidades (Slaughter y Rhoades, 2004).

En años recientes el Ac ha ido adquiriendo connotaciones adicionales, dependiendo de la aproximación utilizada por diversos autores, su lenguaje disciplinario, unidades investigadas y los conceptos asociados a cada una de esas aproximaciones. Un grupo de éstas se focaliza en la investigación que desarrollan las universidades bajo el supuesto de que esta función sirve como plataforma preferente para el avance del AC. Así, Münch (2014) define al AC como un híbrido que reúne la búsqueda científica de la verdad con la maximización de ganancias económicas, tornando a las universidades en empresas que compiten por la acumulación de capital que persiguen nuevos descubrimientos para convertirlos en patentes y mercancías transables.

Otro grupo de aproximaciones se articula a partir de la variada literatura de la universidad emprendedora o empresarialización de la educación superior. Actualmente hay cuatro líneas de desarrollo de esta 
perspectiva: Primero, aquella reflejada en la narrativa positiva de integración de la universidad a una triple hélice, donde interactúa con el sector privado y el gobierno (Etzkowitz y Zhou, 2017), transformándose en la base de una economía o una sociedad basada en el conocimiento; Segundo, una línea que destaca y celebra el despliegue de nuevas capacidades organizacionales y de respuesta institucional a entornos turbulentos y desafiantes (Clark, 2004), dando lugar a la imagen de una organización innovadora capaz de adaptarse a los rápidos y profundos cambios de su medio ambiente; Tercero, una línea que adopta una visión crítica de la universidad emprendedora, interpretándola como un producto de la ideología y economía política neoliberales (Jessop, 2017), entregada al juego de los mercados y creadora del fenómeno del gerencialismo (managerialism) (Deem, 2017). Por último, un cuarto tipo de aproximación se despliega bajo la noción de teoría del AC transnacional (Kauppinen y Cantwell, 2014a). Su principal diferencia con la teoría convencional del Ac es que mientras ésta limita su foco al Estado-nación (Buckner, 2016), la otra, en cambio, asume que en la nueva economía las redes de poder y saber se desplazan hacia el polo global (Kauppinen y Cantwell, 2014b; Kehm, 2014; Knight, 2015).

La intensa actividad que se desarrolla en torno al estudio del capitalismo académico, a pesar del origen definidamente estadounidense de la teoría del AC, ha impulsado su difusión hacia otras latitudes: hacia el resto de los países anglosajones primero, Europa continental luego, países de Europa Central y del Este enseguida y luego hacia América Latina, Asia, países árabes y africanos. En todas partes, las correas de transmisión del AC y su núcleo de ideas parecen ser las mismas: idioma inglés, redes de coautoría entre académicos involucrados con el tema, revistas indexadas, congresos científicos, movilidad de docentes e investigadores, y programas de doctorado de países del Norte donde concurren estudiantes del Sur para realizar sus tesis sobre el AC en sus respectivos países o regiones. De manera tal que el AC, a pesar de ser un concepto esencialmente estadounidense, circula ahora más ampliamente por redes académicas transnacionales.

\section{Metodología}

Para el presente estudio se aplicó el programa Publish or Perish con el fin de determinar los documentos registrados por Google Scholar que en el título contuvieran las palabras "academic capitalism" en inglés, español y portugués, respectivamente. El uso de Google Scholar se justifica, pues permite alcanzar la máxima cobertura posible de documentos académicos en una amplia gama de soportes, incluyendo artículos de revistas, capítulos de libros, libros, ponencias en congresos, tesis y literatura gris (Harzing y Van der Wal, 2008).

Las búsquedas fueron realizadas durante la segunda quincena del mes de mayo de 2018 y arrojaron listas de 368 textos en inglés y 76 en español y portugués, respectivamente; dichos listados fueron depurados, fusionando entradas duplicadas, corrigiendo autores o títulos mal identificados y eliminando entradas en idiomas distintos al inglés, español y portugués.

En idioma inglés el corpus se restringe a los documentos incluidos dentro del índice $\mathrm{H}$ de dicha base de documentos; esto es, aquellos con 33 citas o más, siguiendo el procedimiento especificado por Martínez, Негrera, López-Gijón y Herrera-Viedma (2014) para el estudio de autores que denominan H-clásicos en una determinada área de especialidad. Se agregaron a esta primera colección los demás documentos pertenecientes a autores incluidos en la selección previa, publicados dentro del último quinquenio (20132018) con un número menor de citas que el respectivo índice $H$, con el objeto de incluir en el estudio su producción más reciente. Con esto, la muestra en idioma inglés se incrementó a 41 documentos. Para los documentos en español y portugués se siguió un procedimiento diferente, puesto que el índice H de este corpus resultó bajo (8 y 2 para las bases en español y portugués, respectivamente ${ }^{2}$ ), se incluyó adicionalmente a todos los autores cuyos documentos hubiesen sido citados al menos 1 vez, resultando un total de 23 documentos en ambos idiomas.

De esta manera, el listado de documentos seleccionados inicialmente quedó circunscrito a 41 publicaciones en inglés y 23 en español y portugués. De ellas, se excluyeron las que no contaban con

\footnotetext{
${ }^{2}$ El valor 8 de la base en español representa el índice $\mathrm{H}$ de dicha muestra para la búsqueda realizada durante la segunda quincena del mes de mayo de 2018. Puesto que los documentos en portugués fueron buscados en la segunda quincena de julio del mismo año, el valor 2 refleja su índice $\mathrm{H}$ en esta última fecha.
} 
versión digital y aquellas que carecían de resumen, introducción o conclusión, elementos cruciales para su posterior revisión y análisis. De forma tal que la base final quedó integrada por 33 documentos en inglés y 14 en español y portugués (ver Anexo).

\subsection{Características conceptuales de los documentos}

El análisis de los documentos utiliza dos aproximaciones: la identificación de las características conceptuales de cada documento, por un lado, y el análisis del contenido de los textos, por otro.

Para efecto del análisis conceptual se parte determinando el enfoque utilizado por cada documento. Siguiendo los meta-análisis de Tight (2012) sobre producción de artículos en el campo de estudios de la educación superior, se entiende aquí por enfoque las suposiciones utilizadas para explicar el objeto de interés de cada documento; en este caso, la teoría del Ac. De acuerdo con el mismo autor, se distinguen tres niveles de involucramiento con ese aparato conceptual: 1) nulo (la presentación y discusión de resultados no discute conceptos ni propone una reformulación teórica de manera explícita), 2) superficial (si bien algunos conceptos, modelos y paradigmas pueden ser aplicados, la elección explícita de una teoría está ausente) y 3) completa (un enfoque específico es seleccionado y su elección es fundamentada y los resultados son interpretados a la luz de sus principios).

En segundo lugar, de nuevo bajo la guía de Tight (2012), se determina la orientación del documento. En este ámbito se consideran tres posibilidades: 1) empírica (el documento posee una orientación descriptiva, si bien no involucra necesariamente un apartado de metodología), 2) combinada (el documento utiliza conceptos para analizar su material empírico, los cuales son presentados explícitamente junto a los procedimientos utilizados para su generación) y 3) conceptual (el documento puede o no utilizar referencias empíricas, pero ellas no son un resultado de la investigación y, en cambio, dependen de otros estudios).

En tercer lugar, se identifica su metodología. Ocho alternativas fueron consideradas (Tight, 2012): 1) análisis documental, 2) análisis comparativo, 3) entrevistas, 4) encuesta y análisis multivariados, 5) análisis conceptual, 6) fenomenografía, 7) perspectivas críticas/feministas y 8) estudios biográficos.

Finalmente, se define la unidad de referencia de la investigación (Tight, 2012), que admite nueve posibilidades: 1) individuo, 2) curso, 3) facultad, 4) departamento, 5) disciplina, 6) institución o 7) sistema nacional, 8) regional o 9) mundial. Si la unidad de análisis no es mencionada -por ejemplo, en el caso de estudios sobre la esencia de la universidad- se necesitó otra categoría, "idea de universidad" (10).

\subsection{Análisis del contenido de los documentos}

En un segundo momento, los documentos fueron examinados a través del método de análisis de contenido, que procura generar conocimiento mediante el examen e identificación de patrones, temas y significados pertinentes al cumplimiento de objetivos de la investigación. De acuerdo con Guest, Namey y Mitchell (2013), es posible distinguir dos técnicas de análisis de contenido: guiada por hipótesis o bien por los contenidos. El presente estudio utiliza una opción intermedia. Basado en el análisis cuantitativo y en los temas principales que aparecen como resultado del análisis conceptual, se presta atención a tres diferencias en la utilización del enfoque del Ac en el mundo angloparlante y en América Latina: a) si acaso se halla referido a investigación o a docencia y enseñanza, b) si se centra en procesos de innovación y economía del conocimiento o en capital humano y economía de retornos individuales y, finalmente, c) si el análisis procura una comprensión sociológica del fenómeno o su cuestinamiento crítico. Estas dimensiones son utilizadas a continuación para describir las diferencias entre el uso de la teoría del Ac en inglés y español y portugués.

\section{Resultados}

\subsection{Análisis de las características conceptuales de los documentos}

El análisis de características conceptuales de los documentos sugiere la existencia de claras diferencias en 
la utilización del enfoque del Ac en las investigaciones en inglés y en español y portugués. En primer lugar, existen diferencias en lo relativo al tipo de documentos. En ambos casos, la casi totalidad de documentos corresponde a artículos en revistas indexadas: $82 \%$ para la base en inglés y $71 \%$ para la base en español y portugués. Sin embargo, mientras la base en inglés está conformada, además, por libros y capítulos de libros (18\%), en la base en español y portugués las conferencias y disertaciones poseen una importancia mayor (21\%) que los libros o capítulos de libros (7\%).

El promedio de veces que los textos de cada base de datos son citados es también diferente. Mientras los textos en inglés tienen un número de citaciones promedio de 225 , en el caso de los textos en español y portugués este número es sólo 14. Esta diferencia se explica en medida importante por la opción de considerar sólo documentos con mayor número de citas en la muestra en inglés, mientras que la muestra en español y portugués incluye todos los documentos con al menos una cita. Sin embargo, aun si se consideran todos los documentos que hubiesen sido citados al menos una vez en ambas muestras, y excluyendo los textos fundacionales -altamente citados- de la teoría del AC, la diferencia es sustancial: 50 para 99 textos en inglés y 10 para 32 textos en español y portugués.

Al mismo tiempo, la naturaleza teórica de la reflexión expresada en los textos de ambas muestras es diferente. La totalidad de los documentos en inglés posee un involucramiento completo o superficial con la teoría del AC; esto contrasta con los textos en español y portugués, donde sólo un 36\% menciona explícitamente dicho enfoque o discute sus características. Lo anterior redunda en una diferencia importante en la contribución al desarrollo de la teoría del Ac. Mientras los intentos de revisión y reformulación de la teoría son recurrentes entre los textos en inglés, en los textos en español y portugués reflexiones de esta naturaleza son escasas.

Esto afecta asimismo la relación entre teoría y metodología en los documentos que integran ambas muestras. Mientras $85 \%$ de los textos en inglés utiliza la teoría para analizar un referente empírico definido explícitamente, sólo $29 \%$ de los textos en español y portugués hace lo mismo, optando -en cambio- por promover reflexiones críticas de naturaleza general acerca de los sistemas de educación superior. También la unidad de análisis presenta diferencias significativas. En general, los estudios en inglés se concentran en las universidades de países desarrollados, como Estados Unidos (61\%), otros países angloparlantes desarrollados (3\%), países europeos continentales (12\%) y otros del resto del mundo, incluyendo China y Kenia (6\%). América Latina, como región, o los países de la región no reciben mención alguna en la literatura en inglés. En contraste, las investigaciones en español y portugués se enfocan en sistemas de educación superior de países en desarrollo como México (28.6\%), Chile (14\%) y Brasil (14\%).

\subsection{Análisis de contenido de los documentos}

En relación con el contenido de los textos, se aprecia una clara diferencia en cuanto a la atención prestada a la función de investigación de las universidades, dependiendo de si se trata de la literatura del AC en inglés o en español y portugués. Los textos en inglés enfatizan el hecho que la emergencia del régimen del AC transforma la función de investigación, orientándola hacia la satisfacción de necesidades económicas. En cambio, la utilización del enfoque del Ac en América Latina no presta mayor atención a las nuevas funciones económicas de la investigación, si no que se concentra en el ámbito de la enseñanza, especialmente en la pérdida del potencial crítico de la formación universitaria. Los siguientes fragmentos ilustran esta bifurcación.

These changes represent a profound shift in the way university research is expected to contribute to the public good. Because university administrators are responsible for creating organizational policies and infrastructures that are consistent with their organizational mission and with federal and state laws, it is critical to analyze how university administrators assess university-industry relationships in relation to public- interest scientific research. (Glenna, Lacy, Welsh y Biscotti, 2007, p. 141).

Utilizando el concepto de "capitalismo académico", este artículo examina la neoliberalización de los sistemas educativos europeos y cómo se traduce en la forma en que se imparte y se experimenta la educación universitaria. Sostengo que los cambios descritos anteriormente 
producen una serie de características institucionales poco saludables o, en el contexto de mi argumento, pecados. (Briggs, 2017, p. 1).

Otra diferencia según la filiación idiomática de los documentos corresponde al énfasis puesto en los efectos sociales o individuales del cambio del régimen de conocimiento que traería consigo el Ac. Así, la literatura en inglés subraya la importancia del cambio en términos de "economía del conocimiento" y su impacto en la innovación, mientras que la literatura en español y portugués enfatiza la centralidad de la nueva "economía de retornos individuales", basada en la adquisición de capital humano por parte de los estudiantes. Según puede apreciarse en los siguientes fragmentos, los textos en inglés asignan especial importancia al tránsito desde una sociedad industrial a una cuyo principal medio de producción es el conocimiento especializado, en tanto la literatura en español y portugués asigna relevancia a la visión de la universidad como entidad creadora de capital humano.

While economics and business administration produce economically useful knowledge - at least in a symbolic sense, though not always in reality — sociology and political science are still split into a humanities section, producing cultural capital, and a science section, producing economic capital of instrumentally applicable knowledge and capacities. In the so-called knowledge society, economic capital has gained in value, while cultural capital has decreased in value. This means that degrees in the sciences are more effective than degrees in humanities to obtain access to powerful positions in the economic or political fields of society. (Münch, 2014, p. 8).

Estos elementos permiten la obtención de un cuadro relativo a decisiones con una orientación eminentemente rentista, en la medida en que los esfuerzos están orientados hacia la comercialización de productos (credenciales) no acumulables de manera endógena por las instituciones. Vale decir, la formación de profesionales y técnicos para el pregrado supone la producción (en este caso a escala ampliada) de credenciales cuyos retornos son aprovechados de manera privada por los estudiantes una vez terminados sus estudios y por las empresas al contratar fuerza de trabajo calificada. (Fernández, 2011, pp. 42-43).

Una última distinción según el carácter idiomático de las muestras documentales seleccionadas tiene que ver con los objetivos latentes. Con mayor o menor intensidad, los textos en inglés reconocen que la aproximación desarrollada en Slaughter y Leslie (1997) y Slaughter y Rhoades (2004) representa un intento por comprender transformaciones recientes de los sistemas de educación superior. Independientemente de si se otorga el estatuto de teoría a este enfoque -y esta es una materia sujeta a debate en los mismos textos (Kauppinen, 2013)- el concepto de AC se utiliza como un medio explicativo, capaz de dar cuenta del cambio en el funcionamiento de las universidades contemporáneas.

Diferente es la situación de la literatura en español y portugués. Si bien se encuentran esfuerzos aislados de comprensión teórica del fenómeno del capitalismo académico (Corral, 2015; Fernández, 2009; Fioreze, 2017) la mayor parte de los textos en estos dos idiomas utiliza los conceptos de la teoría del AC para cuestionar los efectos supuestamente perversos de dicho régimen de conocimiento y aprendizaje, o sea, como una modalidad de "problematización epistémica", según denominan a esta práctica GuzmánValenzuela y Barnett (2019). El empleo de esta aproximación adopta aquí una postura "comprometida" mediante la cual se espera modificar y superar el estado de cosas desaprobado. Los dos párrafos siguiente ilustran esta distinción:

What makes the academic capitalism theory useful for this article is its critique of the notion that federal intellectual property policies, private research funding, and other pressures external to the public research organization alone are responsible for the commercialization trend. (...) We believe that this theory of academic capitalism might better explain the commercialization of academic research if it were to incorporate a description of how the structural position of university actors, especially university administrators, influences the ways that university actors participate in and make sense of university-industry relationships. (Glenna et al., 2007, p. 143).

No obstante los avances de los estudios de género, las academias persisten como espacios estructuradores y reproductores de las desigualdades de género, donde las mujeres, actualmente, siguen ocupando un lugar marginal dentro del campo de saber/poder que son las 
universidades. Frente a esta realidad, en el presente artículo consideramos que al estudiar el capitalismo académico desde una concepción de género como la definida, buscamos promover un cambio en esa realidad social de las academias. (De Armas y Venegas, 2016, p. 58).

\section{Discusión}

Para efectos de este estudio, ¿cuál es entonces el conglomerado conceptual que bajo el rótulo de teoría del AC se ha elaborado en el centro y es recibido en América Latina?

Representado numéricamente según la muestra seleccionada de la literatura en inglés, aquel conglomerado se conforma de 33 documentos con un impacto relativamente alto, mientras en el polo de la recepción encontramos 14 documentos de impacto relativamente bajo. De manera que hasta el momento, el AC es una teoría que se produce y lee en inglés y contiene, por lo mismo, un conjunto de visiones, presupuestos, creencias y juicios culturalmente condicionados que no se transmiten fácilmente a otras culturas. Se constata, además, que la literatura en inglés se encuentra organizada más formalmente desde el punto de vista de la comunicación académica -artículos, libros y capítulos de libros- mientras que en español y portugués la literatura gris cobra mayor importancia.

De particular significado para caracterizar el proceso de recepción de esta teoría en América Latina es el hecho de que el involucramiento (engagement) con la reflexión teórica (Tight, 2012) se encuentra casi ausente en los textos sobre capitalismo académico en español y portugués. En cambio, en idioma inglés la situación es diferente, existe un buen número de documentos que elaboran y discuten elementos conceptuales con vistas a la elaboración de la teoría del Ac. Esto tiene consecuencias, además, para la posibilidad de establecer relaciones entre teoría y metodología. Soo unos pocos estudios en español y portugués explicitan este enlace, concentrándose, por el contrario, en el cuestionamiento de los efectos del capitalismo académico.

Adicionalmente, existen diferencias de cobertura geográfica entre ambos grupos. Los estudios en inglés se enfocan en los países angloparlantes (Estados Unidos, Reino Unido, Australia, Canadá y Nueva Zelanda) e incluyen, además, de forma gradual, a otros países europeos, así como a los países BRICs (Brasil, Rusia, India, China y Sudáfrica) y a países individuales de todo el mundo, adquiriendo de esta forma una cobertura global. En cambio, los estudios en español y portugués se concentran en países de la región latinoamericana. Asimismo, se evidencian diferencias temáticas entre ambos grupos de documentos.

Como se evidencia, los estudios en inglés se focalizan en el surgimiento de un régimen capitalista académico de investigación y subrayan los nuevos circuitos de producción, comunicación y valorización del conocimiento al incorporarse éste a los mercados, la actividad empresarial y la economía. Por el contrario, los documentos en español y portugués destacan ante todo un efecto del capitalismo académico, más difuso y de carácter ideológico-político, sobre la educación superior en general, la institución universitaria en particular y las funciones educativas de ésta. En cambio, no llevan a cabo, de manera explícita al menos, una reelaboración o adaptación de conceptos al contexto local, lo que Guzmán-Valenzuela y Barnett (2019) llaman una "matización epistemológica".

Una diferencia adicional observada es que en el polo emisor la teoría es usada con propósitos heurísticos y para guiar estudios empíricos, mientras que en el polo de la recepción se le utiliza primordialmente con propósitos de cuestionamiento, para interrogar críticamente tendencias globales de cambio de la educación superior y políticas en curso en este sector, que amenazarían con transformar y podrían arruinar la esencia de la universidad (Gutierrez, 2018).

De manera que la naturaleza del conglomerado conceptual del AC se altera en el proceso de ser importado desde el centro hacia la periferia del campo de estudio de la educación superior. Al recibirse en América Latina se reifica y adquiere un uso esencialmente descriptivo y de cuestionamiento, perdiéndose el involucramiento con la teoría. De hecho, los documentos en español y portugués mencionan escasamente los términos teoría, marco de referencia, enfoque o paradigma. Según la clasificación de Tight (2012) poseen un involucramiento nulo o, en unos pocos casos, sólo superficial con la teoría. En cambio, comparten con una parte de la investigación contemporánea en este campo la frecuente presencia de una subyacente o explícita crítica del neoliberalismo (Tight, 2019, p. 173). La 
recepción no discute con la teoría del Ac ni la interroga, más bien la celebra y utiliza para efectos de cuestionar sistemas y políticas de educación superior. Incluso en aquellos casos en que se le usa con mayor involucramiento conceptual, como hacen Montes y Mendoza (2018) -ya sea para escudriñar su recepción o aplicarla como marco en una investigación empírica- se conserva parcialmente esa característica de una recepción acrítica.

No debería sorprender, por tanto, que la recepción latinoamericana de la teoría del Ac haya conservado sin mayor problema uno de sus ingredientes más típicamente estadounidenses, esto es, el foco puesto en las universidades públicas de investigación, con su rol de producción de conocimiento. Por el contrario en la región receptora de la teoría hay más de 4,000 instituciones que llevan formalmente el nombre de universidades, la mayoría de las cuales son privadas, con y sin fines de lucro, y tanto entre éstas como entre las universidades públicas sólo una minoría puede clasificarse como research universities (Brunner y Miranda, 2016). El proceso de recepción exhibe a este respecto una de sus mayores debilidades: no sólo el uso preferente y acrítico de teorías generadas en el centro (Guzmán-Valenzuela y Barnett, 2019) sino, además, la conservación de los temas y focos propios de los países de origen y el desconocimiento de características y rasgos idiosincrásicos de la educación superior de la región, como el carácter mixto (público-privado) de la mayoría de los sistemas nacionales, su peculiar economía política, el neto predomino de la escala nacional en los mercados relevantes -de instituciones, estudiantes, personal académico, recursos y prestigios- y las diversas, específicas formas, que adopta la gobernanza de estos sistemas nacionales (Brunner, 2017).

\section{Conclusiones}

Los hallazgos presentados en este artículo pueden resumirse de la siguiente forma: la recepción de la teoría del AC en la literatura en español y portugués ha sido hasta el momento más bien dependiente; esto es, subodinada a la autoridad de su origen, recogiendo sin mayor reflexión y elaboración los presupuestos, las temáticas, los focos y el vocabulario de los países anglosajones, particularmente los Estados Unidos de América, de donde la teoría procede y se importa. En vez de transformarla y enriquecerla, esta modalidad de recepción sirve más bien para cuestionar el presente estado de cosas atribuido a la educación superior latinoamericana y sus políticas, utilizando un vocabulario conceptual que fácilmente puede asimilarse con -y sirve para legitimar- una crítica del neoliberalismo.

Por el contrario, convendría avanzar hacia formas de recepción activa o apropiación creativa (Naidoo, 2016) de la teoría del Ac en la región latinoamericana. Es decir, que permitan explicar mejor las dinámicas estructurales, institucionales y de gobernanza de la educación superior de esta región, incorporando críticamente los aportes de la teoría anglosajona del AC a la vez que reelaborándolos a la luz de las realidades de la región. Con este propósito se ofrecen a continuación tres sugerencias de cara al futuro.

Primero, se requiere revisar minuciosamente las cambiantes constelaciones conceptuales que dan cuenta del capitalismo académico en el polo de la recepción. ¿Cómo interactúan estas constelaciones con la teoría del Ac formulada en el centro? ¿Qué conceptos emplean los procesos de apropiación y cómo se busca estabilizar su significado? ¿Se articulan aquí sólo como un eco de conceptos recibidos desde el centro o hay un involucramiento serio con la teoría y, por ende, una posibilidad de innovación conceptual?

Segundo, se sugiere profundizar el estudio de los usos que se hacen de la teoría del Ac por parte de las comunidades de investigadores latinoamericanos. ¿Cuál relación existe entre teoría, deliberación pública y políticas en el capitalismo académico periférico? ¿Qué usos de la teoría recibida predominan en América Latina y cuáles son los principales actores de la recepción? ¿Cómo opera la apropiación en un campo académico emergente y cuáles son los actores y redes que participan en cada país en la transmisión?

Tercero, es imprescindible elaborar un aparato conceptual y un marco analítico que permitan a la teoría del AC asentar, desde la periferia, una teoría de la variedad del capitalismo académico, teoría que recién comienza a elaborarse en el centro, pero en perspectiva norteamericano-europea (Jessop, 2017). Esto con un doble propósito. Por un lado, avanzar en la caracterización y comprensión de los capitalismos académicos nacionales en su diversidad, sin imponerles como único lente el del polo emisor de la teoría. Por otro lado, estimular estudios comparativos que vayan más allá de casos únicos, contando con un marco de análisis que haga posible comparar la variedad sin sujetarla al canon angloamericano. Desde ya 
contamos con algunos estudios latinoamericanos que apuntan en esa dirección (Brunner, 2017; Fioreze, 2017; Maldonado-Maldonado, 2014; Montes y Mendoza, 2018).

\section{Referencias}

Briggs, D. (2017). Los siete pecados del capitalismo académico y el crimen de la ciencia: Deconstruyendo la ideología de la "ciencia criminológica" en España. Criminología y Justicia Refurbished, 2(3), 1-22.

Brunner, J. J. (2009). Apuntes sobre sociología de la educación superior en contexto internacional, regional y local. Estudios pedagógicos (Valdivia), 35(2), 203-230. Recuperado de

https://dialnet.unirioja.es/servlet/articulo?codigo=3333647

Brunner, J. J. (2017). La economía política del capitalismo académico: el caso de Chile. En H. Lavados y A. L. Durán (Eds.), Desafíos para el desarrollo universitario en Chile (pp. 159-231). Valencia, España: Tirant humanidades.

Brunner, J. J. y Miranda, D. A. (Eds.). (2016). Educación superior en Iberoamérica: Informe 2016. Santiago, Chile: Centro Interuniversitario de Desarrollo.

Brunner, J. J., Labraña, J., Ganga, F. y Rodríguez-Ponce, E. (2019). Circulación y recepción de la teoría del "academic capitalism" en America Latina. Archivos Analíticos de Políticas Educativas, 27(79).

doi:10.14507/epaa.27.4368

Buckner, E. S. (2016). The changing discourse on higher education and the nation-state, 1960-2010. Higher Education, 74(3), 473-489. doi:10.1007/s10734-016-0056-2

Clark, B. R. (2004). Sustaining change in universities: Continuities in case studies and concepts. Nueva York: The Society for Research into Higher Education \& Open University Press.

Corral, S. (2015). El capitalismo académico: gestión del conocimiento y perfil de egreso en la Universidad Autónoma de Ciudad Juárez. Salamanca, España: Universidad Autónoma de Ciudad Juárez. Recuperado de https://gredos.usal.es/handle/10366/128115

De Armas, T. y Venegas, C. (2016). Patriarcado y capitalismo académico. La reproducción de las violencias. En Red Chilena contra la Violencia hacia las Mujeres (Ed.), Educación no sexista. Hacia una real transformación (pp. 57-66). Santiago, Chile: Red Chilena contra la Violencia hacia las Mujeres.

Deem, R. (2017). New managerialism in higher education. En P. Teixeira y J. C. Shin (Eds.), Encyclopedia of international higher education systems and institutions. Dordrecht, Países Bajos: Springer.

Etzkowitz, H. y Zhou, C. (2017). Hélice Tríplice: Inovação e empreendedorismo universidade-indústriagoverno [Triple hélice: innovación y emprendimiento universidad-industria-gobierno]. Estudos Avançados, 31(90), 23-48. doi:10.1590/s0103-40142017.3190003

Fernández, E. (2009). El sistema-mundo del capitalismo académico: procesos de consolidación de la universidad emprendedora. Education Policy Analysis Archives, 17(21), 1-43.

doi:10.14507/epaa.v17n21.2009

Fernández, R. (2011). Capitalismo académico en la Educación Superior Chilena. ¿Modelo exitoso para el desarrollo de la ciencia? Análisis de las tendencias en la productividad científica en investigación durante la última década. Science for Innovation, 1(1), 36-45.

Fioreze, C. (2017). O modelo comunitário de universidade e o tensionamento público-privado: entre o capitalismo acadêmico e o compromisso social [El modelo de universidad comunitaria y la tensión públicoprivada: entre el capitalismo académico y el compromiso social]. (Tesis doctoral). Porto Alegre, Brasil: Universidade Federal Do Rio Grande Do Sul. Recuperado de https://lume.ufrgs.br/handle/10183/159149 
Glenna, L., Lacy, W., Welsh, R. y Biscotti, D. (2007). University administrators, agricultural biotechnology, and academic capitalism: Defining the public good to promote university-industry relationships. The Sociological Quarterly, 48(1), 141-163. doi:10.1111/j.1533-8525.2007.00074.x

Guest, G., Namey, E. y Mitchell, M. (2013). Collecting qualitative data: A field manual for applied research. Washington DC: Sage.

Gutierrez, F. P. (2018). Universidad Pública S.A.: Necolonialismo Extractivista Del Conocimiento Científico En La Época Del Capitalismo Cognitivo. Revista FAIA, 7(31), 1-31. Recuperado de http://editorialabiertafaia.com/pifilojs/index.php/FAIA/article/view/154

Guzmán-Valenzuela, C. y Gómez, C. (2019). Advancing a knowledge ecology: Changing patterns of higher education studies in Latin America. Higher Education, 77(1), 115-133. doi:10.1007/s10734-018-0264-z

Guzmán-Valenzuela, C. y Barnett, R. (2019). Patterns of theory use in qualitative research in higher education studies in Latin America: a geopolitical interpretation. International Journal of Qualitative Studies in Education, 32(5), 477-492. doi:10.1080/09518398.2019.1597213

Harzing, A.-W. y van der Wal, R. (2008). Google Scholar as a new source for citation analysis. Ethics in Science and Environmental Politics, 8(1), 61-73. doi:10.3354/esep00076

Jessop, B. (2017). Varieties of academic capitalism and entrepreneurial universities. Higher Education, 73(6), 853-870. doi:10.1007/s10734-017-0120-6

Kauppinen, I. (2013). Towards a theory of transnational academic capitalism. British Journal of Sociology of Education, 36(2), 336-353. doi:10.1080/01425692.2013.823833

Kauppinen, I. y Cantwell, B. (2014a). The global enterprise of higher education. In B. Cantwell, I. Kauppinen y S. Slaughter (Eds.), Academic capitalism in the age of globalization (pp. 137-146). Baltimore: Johns Hopkins University Press.

Kauppinen, I. y Cantwell, B. (2014b). Transnationalization of academic capitalism through global production networks. En B. Cantwell y I. Kauppinen (Eds.), Academic capitalism in the age (pp. 147-165). Baltimore: Johns Hopkins University Press. 
Kehm, B. (2014). Global university rankings - Impacts and unintended side effects. European Journal of Education, 49(1), 102-112. doi:10.1111/ejed.12064

Keim, W. (2014). Conceptualizing circulation of knowledge in the social sciences. En W. Keim et al. (Eds.), Global knowledge in the social sciences. Made in circulation (pp. 87-113). Inglaterra: Ashgat.

Knight, J. (2015). International universities misunderstandings and emerging models? Journal of Studies in International Education, 19(2), 107-121. doi:10.1177/1028315315572899

Maldonado-Maldonado, A. (2014). Peripheral knowledge-driven economies: What does academic capitalism have to say? In B. Cantwell y I. Kauppinen (Eds.), Academic capitalism in the age of globalization (pp. 187-207). Baltimore: Johns Hopkins University Press.

Martínez, M. A., Herrera, M., López-Gijón, J. y Herrera-Viedma, E. (2014). H-Classics: Characterizing the concept of citation classics through H-index. Scientometrics, 98(3), 1971-1983. doi:10.1007/s11192-0131155-9

Montes, I. C. y Mendoza, P. (2018). Docencia e investigación en Colombia desde la perspectiva del capitalismo académico. Education Policy Analysis Archives, 26(40), 1-33. doi:10.14507/epaa.26.3220

Münch, R. (2014). Academic capitalism: Universities in the global struggle for excellence. Nueva York: Routledge.

Naidoo, K. (2016). Against theoretical evangelism: Imagining the possibilities of a critical approach to theorizing in professional academic development. South African Journal of Higher Education, 30(6), 8-23. doi:10.20853/30-6-715

Ordorika I. y Rodríguez-Gómez R. (2018). Field of higher education research in Latin America. En P. Teixeira y J. Shin (Eds.), Encyclopedia of International Higher Education Systems and Institutions. Países Bajos: Springer.

Slaughter, S. y Leslie, L. (1997). Academic capitalism: Politics, policies, and the entrepreneurial university. Baltimore: Johns Hopkins University Press.

Slaughter, S. y Rhoades, G. (2004). Academic capitalism and the new economy: Markets, state, and higher education. Battimore: Johns Hopkins University Press.

Tight, M. (2012). Researching higher education. Berkshire, Inglaterra: Society for Research into Higher Education \& Open University Press.

Tight, M. (2019). Higher Education Research. The Developing Field. Londres-Nueva York: Bloomsbury Academic. 


\section{Anexo. Artículos consultados}

\begin{tabular}{|c|c|c|}
\hline \multicolumn{3}{|c|}{ Referencias de documentos utilizados en inglés } \\
\hline Autor (es) & $\begin{array}{c}\text { Año de } \\
\text { publicación }\end{array}$ & Título \\
\hline Choi, P. K. & 2010 & $\begin{array}{l}\text { - 'Weep for Chinese university': A case study of English hegemony and } \\
\text { academic capitalism in higher education in Hong Kong. }\end{array}$ \\
\hline Deem, R. & 2001 & $\begin{array}{l}\text { - Globalisation, new managerialism, academic capitalism and } \\
\text { entrepreneurialism in universities: Is the local dimension still } \\
\text { important? }\end{array}$ \\
\hline Enders, J. & 2001 & $\begin{array}{l}\text { - Between state control and academic capitalism: A comparative } \\
\text { perspective on academic staff in Europe. }\end{array}$ \\
\hline $\begin{array}{l}\text { Glenna, L., Lacy, W., Welsh, R., y } \\
\text { Biscotti, D. }\end{array}$ & 2007 & $\begin{array}{l}\text { - University administrators, agricultural biotechnology, and academic } \\
\text { capitalism: Defining the public good to promote university-industry } \\
\text { relationships. }\end{array}$ \\
\hline $\begin{array}{l}\text { Gonzales, L. D., Martinez, E., y } \\
\text { Ordu, C. }\end{array}$ & 2013 & $\begin{array}{l}\text { - Exploring faculty experiences in a striving university through the } \\
\text { lens of academic capitalism. }\end{array}$ \\
\hline Hanley, L. & 2005 & - $\quad$ Academic Capitalism in the new university. \\
\hline Hoffman, S. G. & 2011 & $\begin{array}{l}\text { - The new tools of the science trade: Contested knowledge } \\
\text { production and the conceptual vocabularies of academic capitalism. }\end{array}$ \\
\hline Johnson, A. T., y Hirt, J. B. & 2011 & $\begin{array}{l}\text { - Reshaping academic capitalism to meet development priorities: The } \\
\text { case of public universities in Kenya. }\end{array}$ \\
\hline Kauppinen, I. & $\begin{array}{l}2012 \\
2013 \\
2013\end{array}$ & $\begin{array}{l}\text { - } \quad \text { Towards transnational academic capitalism. } \\
\text { - } \quad \text { Academic capitalism and the informational fraction of the } \\
\text { transnational capitalist class. } \\
\text { - Towards a theory of transnational academic capitalism. }\end{array}$ \\
\hline Kauppinen, I., y Kaidesoja, T. & 2014 & - $\quad$ A shift towards academic capitalism in Finland. \\
\hline $\begin{array}{l}\text { Leslie, L., Rhoades, G., y Oaxaca, } \\
\text { R. }\end{array}$ & 1999 & - Technology transfer and academic capitalism. \\
\hline Mars, M. M., y Rhoades, G. & 2012 & $\begin{array}{l}\text { - Socially oriented student entrepreneurship: A study of student } \\
\text { change agency in the academic capitalism context. }\end{array}$ \\
\hline Mendoza, P. & 2012 & $\begin{array}{l}\text { - The role of context in academic capitalism: The industry-friendly } \\
\text { department case. } \\
\text { - Academic capitalism and doctoral student socialization: A case } \\
\text { study. }\end{array}$ \\
\hline Mendoza, P., y Berger, J. & 2008 & - $\quad$ Academic capitalism and academic culture: A case study. \\
\hline $\begin{array}{l}\text { Mendoza, P., Kuntz, A. M., y } \\
\text { Berger, J. B. }\end{array}$ & 2016 & $\begin{array}{l}\text { - Bourdieu and academic capitalism: Faculty "habitus" in materials } \\
\text { science and engineering. }\end{array}$ \\
\hline Metcalfe, A. S. & 2016 & - Revisiting Academic Capitalism in Canada: No longer the exception. \\
\hline Münch, R. & 2014 & $\begin{array}{l}\text { - Academic Capitalism: Universities in the global struggle for } \\
\text { excellence. }\end{array}$ \\
\hline Paasi, A. & $\begin{array}{l}2013 \\
2015 \\
2016\end{array}$ & $\begin{array}{l}\text { - } \quad \text { Fennia: Positioning a peripheral but international journal under the } \\
\text { - } \quad \text { Acandition of 'academic capitalism'. } \\
\text { - } \quad \text { Globalisation, academic capitalism, and the uneven geographies of } \\
\text { international journal publishing spaces. }\end{array}$ \\
\hline Renault, C. S. & 2006 & $\begin{array}{l}\text { - Academic capitalism and university incentives for faculty } \\
\text { entrepreneurship. }\end{array}$ \\
\hline Rhoades, G. & 2005 & - $\quad$ Capitalism, academic style, and shared governance. \\
\hline Rhoades, G., y Slaughter, S. & 1997 & $\begin{array}{l}\text { - } \quad \text { Academic capitalism, managed professionals, and supply-side higher } \\
\text { education. } \\
\text { - } \quad \text { Academic capitalism in the new economy: Challenges and choices. }\end{array}$ \\
\hline Rhoades, G., y Torres-Olave, B. & 2015 & $\begin{array}{l}\text { - Academic capitalism and (secondary) academic labor markets: } \\
\text { Negotiating a new academy and research agenda. }\end{array}$ \\
\hline Slaughter, S., y Leslie, L. L. & 2001 & - Expanding and elaborating the concept of academic capitalism. \\
\hline Slaughter, S., y Rhoades, G. & 2004 & $\begin{array}{l}\text { - Academic capitalism and the new economy: Markets, state, and } \\
\text { higher education. }\end{array}$ \\
\hline $\begin{array}{l}\text { Welsh, R., Glenna, L., Lacy, W., y } \\
\text { Biscotti, D. }\end{array}$ & 2008 & $\begin{array}{l}\text { - Close enough but not too far: Assessing the effects of university- } \\
\text { industry research relationships and the rise of academic capitalism. }\end{array}$ \\
\hline Ylijoki, O.-H. & 2003 & $\begin{array}{l}\text { - Entangled in academic capitalism? A case-study on changing ideals } \\
\text { and practices of university research. }\end{array}$ \\
\hline
\end{tabular}




\begin{tabular}{|c|c|c|}
\hline \multicolumn{3}{|c|}{ Referencias de documentos utilizados en castellano y portugués } \\
\hline Autor (es) & $\begin{array}{c}\text { Año de } \\
\text { publicación }\end{array}$ & Título \\
\hline Armas, T. de y Venegas, C. & 2016 & - $\quad$ Patriarcado y capitalismo académico. La reproducción de las violencias. \\
\hline $\begin{array}{l}\text { Azevedo, Mário Luiz Neves } \\
\text { de }\end{array}$ & 2015 & $\begin{array}{l}\text { - } \quad \text { Transnacionalização e mercadorização da educação superior: } \\
\text { Examinando alguns efeitos colaterais do capitalismo acadêmico (sem } \\
\text { riscos) no Brasil - A expansão privado-mercantil }\end{array}$ \\
\hline Briggs, D. & 2017 & $\begin{array}{l}\text { - Los siete pecados del capitalismo académico y el crimen de la ciencia: } \\
\text { Deconstruyendo la ideología de la "ciencia criminológica" en España. }\end{array}$ \\
\hline Corral, S. & 2015 & $\begin{array}{l}\text { - El capitalismo académico: gestión del conocimiento y perfil de egreso en } \\
\text { la Universidad Autónoma de Ciudad Juárez. }\end{array}$ \\
\hline Díez, E. & 2009 & $\begin{array}{l}\text { - } \quad \text { El capitalismo académico: La reforma universitaria europea en el } \\
\text { contexto de la globalización. } \\
\text { - } \quad \text { El Plan Bolonia. Capitalismo académico superior. } \\
\text { - El capitalismo académico y el Plan Bolonia. }\end{array}$ \\
\hline Fernández, E. & 2009 & $\begin{array}{l}\text { - El sistema-mundo del capitalismo académico: procesos de consolidación } \\
\text { de la universidad emprendedora. }\end{array}$ \\
\hline $\begin{array}{l}\text { Fernández, E., Rodríguez, } \\
\text { H. y Rodríguez, M. }\end{array}$ & 2010 & $\begin{array}{l}\text { - La formación inicial de profesionales de la educación: Un análisis crítico } \\
\text { de los nuevos planes de estudio en el contexto del "capitalismo } \\
\text { académico". }\end{array}$ \\
\hline Fernández, R. & 2011 & $\begin{array}{l}\text { - Capitalismo académico en la Educación Superior Chilena ¿Modelo } \\
\text { exitoso para el desarrollo de la ciencia? Análisis de las tendencias en la } \\
\text { productividad científica en investigación durante la última década. }\end{array}$ \\
\hline Fioreze, C. & 2017 & $\begin{array}{l}\text { - O modelo comunitário de universidade e o tensionamento público- } \\
\text { privado: entre o capitalismo acadêmico e o compromisso social. }\end{array}$ \\
\hline Ibarra, E. & 2005 & 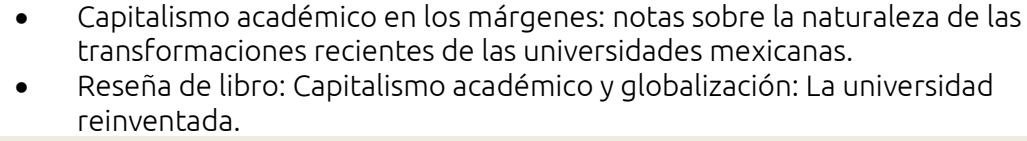 \\
\hline Krotz, E. & 2012 & $\begin{array}{l}\text { - Ciberespacio, ciudadanía, capitalismo académico: Cotidianidad } \\
\text { estudiantil y enseñanza de la Antropología. }\end{array}$ \\
\hline
\end{tabular}

